\section{First case report of Stevens- Johnson syndrome complicated with macrophage activation syndrome}

\author{
Huasong Zeng, Xiangyuan Chen \\ Department of Allergy, Immunology and \\ Rheumatology, Guangzhou Children's \\ Hospital, Guangzhou Medical College, \\ Guangzhou Women's and Children's \\ Medical Center, Guangzhou, Guangdong, \\ China
}

\begin{abstract}
Stevens-Johnson syndrome (SJS) is a complex immunological syndrome with high morbidity and mortality and characterized by acute blistering affecting the skin and at least two mucous membranes. Macrophage activation syndrome (MAS) is a severe, potentially lifethreatening syndrome that often occurs in children and adults with autoimmune disease, especially systemic-onset juvenile idiopathic arthritis or adult-onset Still's disease. This case report highlights the potential risks of SJS developing into MAS, and suggests that rapid diagnosis and treatment are essential to achieving favorable outcomes.
\end{abstract}

\section{Introduction}

Stevens-Johnson syndrome (SJS), also known as erythema multiforme major, ${ }^{1}$ is a serious mucocutaneous illness with systemic symptoms characterized by the presence of flat, atypical target lesions and epidermal detachment encompassing less than $10 \%$ of the total body surface area. Two or more mucosal sites are affected usually. Drugs, infections, and underlying malignancies have been implicated as potential causes of SJS. Macrophage activation syndrome (MAS) is a rare and potentially life-threatening disease. It occurs as a result of immune dysregulation manifested as excessive macrophage proliferation in response to a triggering agent, such as infections, rheumatologic diseases, or tumors. In this report we present the first case of SJS complicated with MAS.

\section{Case Report}

In July, a previously healthy 7-month-old boy was hospitalized with a fever lasting for 20 days and a skin lesion for 15 days. He had a history of a high-grade fever and cough. Ceftriaxone sodium was used as a treatment after a diagnosis of bronchitis was made but without success. After five days, a red rash appeared and spread rapidly across the body. His symptoms were rashes, target-shaped lesions, conjunctival congestion, dry and crustose lips, and dental ulcers. Tienam and a low dose of dexamethasone were used and ceftriaxone sodium discontinued, with no improvement. A blood analysis showed: WBC, 16.0x $10 \%$ L; N, 76.6\%; Hb, $106 \mathrm{~g} / \mathrm{L}$, sedimentation rate (ESR), $43 \mathrm{~mm} / \mathrm{h}$; C-reactive protein (CRP), 37 $\mathrm{mg} / \mathrm{L}$. Liver function was normal, and bone marrow investigation revealed an infection. Stevens-Johnson syndrome was diagnosed and anti-allergic and anti-infective treatment initiated with chlorpheniramine, erythromycin, low-dose dexamethasone, intravenous immunoglobulin (IVIG), and other supportive regimens. The skin and mucosal lesions gradually recovered but the low fever persisted. On the seventh day after admission, the blood culture showed Micrococcus luteus, which was sensitive to vancomycin, given to the patient for three days for the continuous fever. On the eleventh day, bleeding points with splenohepatomegalia were seen. Laboratory tests showed pancytopenia and hepatic dysfunction; elevated levels of bilirubin, triglyceride and serum ferritin; ESR, $2 \mathrm{~mm} / \mathrm{h}$; CRP, $15.24 \mathrm{mg} / \mathrm{L}$. The bone marrow showed a significantly higher proportion of histiocytes and hemophagocytosis. The diagnosis of SJS complicated with MAS was made. Cyclosporine and pulse methylprednisolone were administered. On the fourteenth day, the baby was afebrile and his condition obviously improved. On the twenty-fifth day, laboratory tests were normal and the baby was discharged in good general health.

\section{Discussion}

This is the first report of SJS complicated with MAS. It is important to note that both are potentially fatal diseases. SJS is one of the most severe adverse cutaneous reactions to drugs. It is considered now to be a hypersensitivity disorder, with an occurrence of 1-7 cases per million people per year. Mortality rates in SJS have been reported at about $5-15 \%$ of cases. ${ }^{1}$ There are four etiologic categories: drug-induced, infectious, malignancy-related, and idiopathic. Drugs are the commonest cause of SJS. ${ }^{1,2}$ There are more than 200 medications that have been implicated but common offenders include anti-epileptics, sulphonamide antibiotics, penicillins, allopurinol, and oxicam NSAIDs. ${ }^{2,3}$ The risk was often in the first few weeks of drug intake. ${ }^{3}$ The infectious agents Mycoplasma pneumoniae
Correspondence: Huasong Zeng, 318 Renmin Middle Road, GuangZhou City, Guang Dong Province, China. E-mail: huasongz@gmail.com

Key words: Stevens-Johnson syndrome, macrophage activation syndrome.

Received for publication: 22 April 2009.

Revision received: 7 August 2009.

Accepted for publication: 5 October 2009.

This work is licensed under a Creative Commons Attribution 3.0 License (by-nc 3.0).

(C) Copyright H. Zeng, X. Chen, 2009

Licensee PAGEPress, Italy

Rheumatology Reports 2009; 1:e9

doi:10.4081/rr.2009.e9

have been implicated as a major cause of SJS, others including viral (herpes simplex virus, Epstein-Barr virus), bacterial, fungal, and protozoal causes. ${ }^{1,2,4}$ However, the principal etiology of SJS in children is controversial. Some consider that infections mostly cause $\mathrm{SJS},{ }^{4}$ while others think that drugs are responsible. ${ }^{5}$ Our patient had an infection and had used ceftriaxone sodium, both of which may contribute to SJS. According to the normal etiology, ceftriaxone sodium has the stronger link to SJS. No matter what the etiology is, every patient is unique, and therapy must be administered first according to the individual conditions. Effective management of SJS can decrease mortality. Any suspected causative drug must be stopped immediately and optimal supportive care, which is still the mainstay of treatment in patients with SJS, includes strict isolation, maintenance of fluid and electrolyte homeostasis, prevention and treatment of infections, nutritional support, and adequate analgesia. The role of systemic corticosteroids and IVIG in SJS is controversial. One study compared the use of supportive therapy, IVIG, and corticosteroids. The authors observed no significant benefit from any treatment and concluded that the evidence is insufficient to recommend any specific treatment. ${ }^{6}$ Some studies considered that corticosteroids and IVIG can reduce morbidity and improve patient outcome. Immunosuppressive and immunomodulatory agents, such as cyclosporine, cyclophosphamid, and thalidomide, have been used to treat SJS. However, the efficacy of these agents has not been demonstrated by any controlled clinical trial. Early and effective therapy can curtail the course of SJS and result in a better outcome. However, some complications are inevitable. The short-term complications include skin lesions secondary to the infection, organ dysfunction, and morbidity, as well as long-term complications as a result of mucocutaneous fibrosis and strictures, including ocular lesions and esophageal stricture? 
Our patient had a different complication: MAS.

MAS is a grave disorder with a mortality of about $10-20 \%$. It is characterized by persistent fever, pancytopenia, serious liver disease, acute coagulation abnormalities, and neurological involvement. Several mechanisms inducing MAS have been proposed in association with the underlying disease, and are commonly triggered by infections and medications. However, MAS may occur without any identifiable precipitating factor. ${ }^{8}$ The diagnosis of MAS is established when at least five of the following criteria are met: fever, bicytopenia, hypertriglyceridemia, hemophagocytosis, a serum ferritin level $>500 \mu \mathrm{g} / \mathrm{L}$, splenomegaly, low natural killer cell activity, and increased serum levels of $\operatorname{sCD} 25 .{ }^{9}$ Our case met the first six criteria, with MAS being secondary to SJS. The trigger agent for MAS is not clear but we should pay attention to the influence of vancomycin. Olgar et al. reported four patients with systemic juvenile idiopathic arthritis who developed MAS during or after vancomycin treatment. ${ }^{10}$ Other therapeutic drugs thought to trigger MAS are gold preparations, MTX, sulfasalazine, and TNF-blocking agents. ${ }^{11}$

Treatment of MAS has not been standardized in patients yet, but it commonly includes a variety of agents such as corticosteroids, cyclosporine A, IVIG, etoposide, cyclophophamid, anti-TNF- $\alpha$, methotrexate, G-CSF, and in some cases plasmapheresis. ${ }^{8}$ Our patient's condition changed markedly after the initiation of immunosuppressive treatment with cyclosporine A and methylprednisolone, as we described here. Correct diagnosis and aggressive treatment can result in a favorable outcome.
In conclusion, we report a case of MAS in a seven-month-old boy suffering from SJS. His clinical and laboratory features were typical of SJS and MAS, and his clinical course showed improvement after immunosuppressive therapy with methylprednisolone and cyclosporine A. This highlights the finding that MAS can occur with SJS, and also the potential risk of SJS developing into MAS. Both SJS and MAS are severe, potentially life-threating diseases. It is essential for clinicians to be highly vigilant, to make an early diagnosis, and to start prompt treatment to ensure a successful outcome.

\section{References}

1. Hazin R, Ibrahimi OA, Hazin MI, et al. Stevens-Johnson syndrome: pathogenesis, diagnosis, and management. Ann Med 2008;40:129-38.

2. Koh MJ, Tay YK. An update on StevensJohnson syndrome and toxic epidermal necrolysis in children. Curr Opin Pediatr 2009 Aug;21:505-10.

3. Mockenhaupt M, Viboud C, Dunant A, et al. Stevens-Johnson syndrome and toxic epidermal necrolysis: assessment of medication risks with emphasis on recently marketed drugs. The EuroSCAR-Study. J Invest Dermatol 2008:128:35-44.

4. Léauté-Labrèce $\mathrm{C}$, Lamireau T, Chawki D, et al. Diagnosis, classification, and management of erythema multiforme and Stevens-Johnson syndrome. Arch Dis Child 2008;83:347-52.
5. Levi N, Bastuji-Garin S, Mockenhaupt M, et al. Medications as risk factors of Stevens-Johnson syndrome and toxic epidermal necrolysis in children: a pooled analysis. Pediatrics 2009;123:e297-304.

6. Schneck J, Fagot J, Sekula P, et al. Effects of treatment on the mortality of StevensJohnson syndrome and toxic epidermal necrolysis: a retrospective study on patients included in the prospective EuroSCAR study. J Am Acad Dermatol 2008; 58:33-40.

7. Misra SP, Dwivedi M, Misra V. Esophageal stricture as a late sequel of StevensJohnson syndrome in adults: incidental detection because of foreign body impaction. Gastrointest Endosc 2004;59: 437-40.

8. Tristano AG. Macrophage activation syndrome:A frequent but under-diagnosed complication associated with rheumatic diseases. Med Sci Monit 2008;14:27-36.

9. Emmenegger U, Schaer DJ, Larroche C, et al. Haemophagocytic syndromes in adults: current concepts and challenges ahead. Swiss Med Wkly 2005;135:299-314.

10. Olgar S, Ertugrul T, Devecioglu 0, et al. Does Red-Man reaction stimulate macrophage activation syndrome in children with systemic juvenile idiophathic arthritis? J Rheumatol 2007; 34:2491-4.

11. Grom AA. Natural killer cell dysfunction: A common pathway in systemic-onset juvenile rheumatoid arthritis, macrophage activation syndrome, and hemophagocytic lymphohistiocytosis? Arthritis Rheum 2004;50:689-98. 